\title{
QUALITY OF LIFE AMONGST BENZODIAZEPINE USERS PRE AND POST DEPRESCRIBING
}

\author{
SHILPA SHREE H. S.1, NAVEEN KUMAR N.1, MAHESWARI E.2*, VIRUPAKSHA H. S. ${ }^{3}$
}

${ }^{1}$ Department of Pharmacy Practice, M. S. Ramaiah College of Pharmacy, Bangalore, Karnataka, India, ${ }^{2}$ Department of Pharmacy Practice, Faculty of Pharmacy, M. S. Ramaiah University of Applied Sciences, Bangalore, Karnataka, India, ${ }^{3}$ Department of Psychiatry, Assistant Professor, Ramaiah Medical College and Hospitals, Bangalore, Karnataka, India

Email: maheswari_eswar@yahoo.com

Received: 06 May 2019 Revised and Accepted: 21 Jun 2019

\section{ABSTRACT}

Objective: The study aimed to analyze the Quality of Life (QoL) of patients utilizing benzodiazepines (BZDs).

Methods: A prospective interventional study was conducted among 109 patients who met the inclusion criteria. The patients consuming BZDs more than the prescribed duration were identified and deprescribed. Deprescribing plan was advised after discussing with the prescribing psychiatrist and inappropriate BZD users. The QoL of patient's pre and post-intervention amongst both the continuing and deprescribed groups was evaluated by WHO-QoL BREF questionnaire.

Results: Mean scores in the domains of physical health (71.48), psychological health (68.74), social relationships (65.15) and environmental health (68) among the deprescribed group was higher compared to the maintenance group. Independent $t$-test statistic for QoL showed a statistical significance in all 4 domains with $\mathrm{p}$ value $<0.05$.

Conclusion: The current study emphasizes that deprescribing of BZDs as shared decision model involving the physician, pharmacist and patients improves the QoL of the patient.

Keywords: Life amongst benzodiazepine, Post deprescribing

(C) 2019 The Authors. Published by Innovare Academic Sciences Pvt Ltd. This is an open-access article under the CC BY license (http://creativecommons.org/licenses/by/4.0/) DOI: http://dx.doi.org/10.22159/ijpps.2019v11i8.33960

Deprescribing is a newer concept described as "the process of withdrawal of potentially inappropriate medications, supervised by a health care professional with the goal of improving outcomes by tapering, discontinuing or prescribing medications on SOS basis" [1] Benzodiazepines (BZDs) are widely prescribed in the short-term treatment of anxiety disorders and insomnia. Despite recommendations for transient or short term use i. e for a duration not exceeding 2-4weeks, prolonged use is a widespread phenomenon in medical practice [2]. Chronic BZD use i. e more than 3 months duration may lead to distressing adverse effects such as dependence, tolerance and cognitive impairments $[3,4]$. However, a recent survey conducted in India revealed that patients take medications for longer durations than required due to health literacy issues Kamath et al. (2017) [5]. In consequence, clinical pharmacists and psychiatrists have a vital role to play in educating the patient's on deprescribing practices thereby increase the patient's medication knowledge, promote lifestyle modifications and thus help in enhancing the patients Quality of Life (QoL) [1,5]. The literature reveals that none of the studies are conducted to evaluate the QoL among the inappropriate BZDs users and this is the first study in India that has looked upon assessing the QoL of patients before and after deprescribing BZDs. Henceforth, the present study was designed to examine the effects of deprescribing BZDs on the patient's overall QoL.

A prospective, interventional study was designed and conducted among 109 patients for a period of 7 mo from August 2017 to February 2018 in both the inpatient and outpatient settings of the Department of Psychiatry at Ramaiah Medical Hospital, Bangalore. Study population included all patients above 18yrs who were already prescribed with BZD or Z-drugs. Elderly patients aged above 85yrs, patients on antiepileptic medications for a seizure disorder and patients with a history of alcohol dependence were excluded from the study.

The study protocol was reviewed and approved by the Institutional Ethics Committee. A written informed consent was obtained from participants who fulfilled the eligibility criteria and willing to participate in the study. The study was performed by collecting relevant data from the patient's prescription forms, case records and by interviewing the patients or caretakers in a well-designed patient data collection form. The deprescribing plan was suggested after discussing with the prescribing psychiatrist and inappropriate BZD users. The QoL of patient's before and after deprescribing in both the continuing (maintenance) and deprescribed groups was assessed by the WHO-QoL BREF Questionnaire. The patients were followed up depending on the duration of tapering and discontinuation of BZDs or Z-drugs.

Data were coded and analysed using IBM SPSS Statistics version 21.0 for windows. The categorical variables were presented using frequencies and percentages. Mean scores of each domain in the WHO-QoL Questionnaire i. e physical health, psychological health, social relationships and environmental health was calculated for both the groups. Independent $\mathrm{t}$-test statistic was employed to test the statistical significance in the QoL among both the groups. Pvalue $<0.05$ was considered as statistically significant.

In the present study of the entire study population, $10(9.1 \%)$ subjects were appropriate BZD users (<4weeks duration), 36(33\%) subjects were short term users (<3months) and 63(57.7\%) subjects were long term users (more than 3months). Post-intervention, $13(11.9 \%)$ were lost during follow up, 56(51.37\%) were on maintenance BZDs and 40(36.69\%) were deprescribed of BZDs. Fig. 1 shows the mean scores in all the domains of the WHO-QoL BREF questionnaire for both the groups. Upon calculating the mean scores in all the 4 domains including physical health (71), psychological health (68), social relationships (65) and environmental health (68), it was found that the scores of the patients deprescribed was higher compared to those continuing BZD use.

Table 1 depicts the independent t-test statistic between follow-ups of both the groups. On applying the Independent $t$-test statistic for Pre-Deprescribing (follow up) and Post deprescribing (follow up) it was found that there was a statistical significance between the two groups in all 4 domains with the $p$ value $<0.05$. This implies that 
deprescribing has a positive impact on the patient's overall QoL. This finding is similar to another review which observed a decline in QoL in patients continuing BZDs than those who discontinued [6]. The drawback of this study included lesser sample size and short follow up periods. The literature on the QoL analysis amongst the BZDs users is still poor. Henceforth, further studies are needed to improve treatment strategies and prevent to potential drug-related problems. Implementing deprescribing practice among the inappropriate BZD users will help to improve the patient's overall QoL.

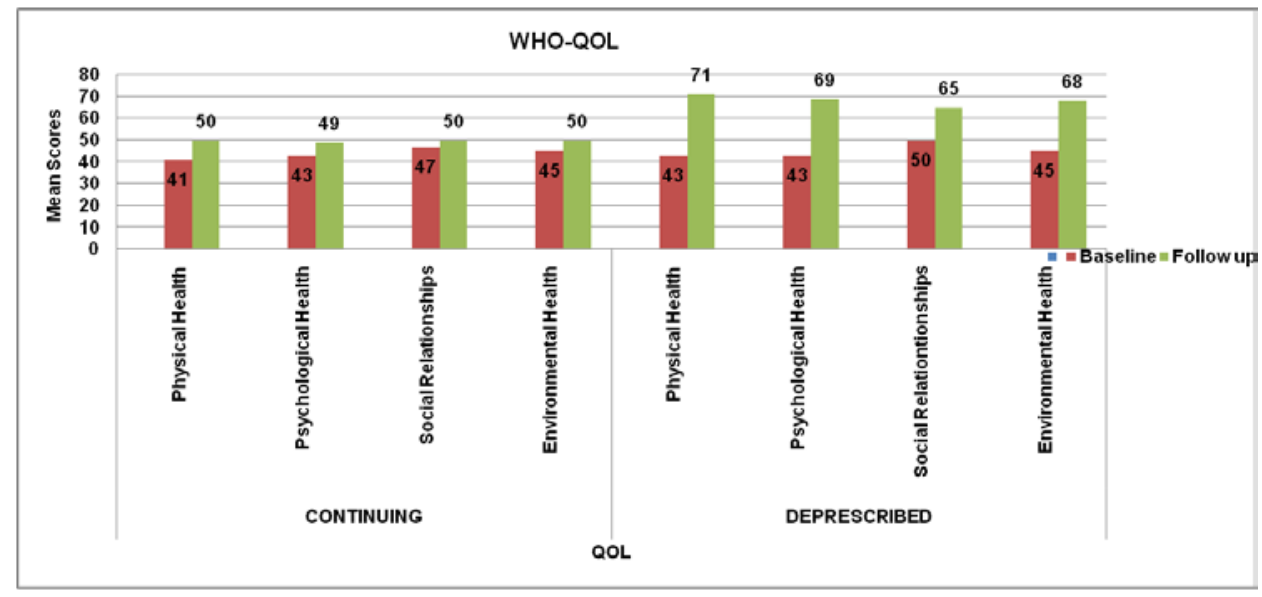

Fig. 1: Quality of life assessment pre and post intervention amongst both the groups

Table 1: Independent t test statistic for assessing quality of life post intervention amongst both the groups

\begin{tabular}{|c|c|c|c|}
\hline Groups & Domains & t-value & Significance (2tailed) \\
\hline \multirow[t]{4}{*}{ Maintenance (Follow up) Vs Deprescribed (Follow Up) } & Domain 1 (Physical Health) & 10.67545 & $<0.005$ \\
\hline & Domain 2 (Psychological Health) & 10.48822 & $<0.005$ \\
\hline & Domain 3 (Social Relationships) & 8.65475 & $<0.005$ \\
\hline & Domain 4 (Environmental Health) & 13.5481 & $<0.005$ \\
\hline
\end{tabular}

\section{AUTHORS CONTRIBUTIONS}

All the author have contributed equally

\section{CONFLICT OF INTERESTS}

Declared none

\section{REFERENCES}

1. Reeve E, Wiese MD. Benefits of deprescribing on patient's adherence to medications. Int J Clin Pharm 2014;36:26-9.

2. Hulten R, Bakker AB, Lodder AC, Teeuw KB, Bakker A, Leufkens HG. The impact of attitudes and beliefs on length of benzodiazepine use: a study among inexperienced and experienced benzodiazepine users. Soc Sci Med 2003;56:134554 .

3. Xiang YT, Weng YZ, Leung CM, Tang WK, Ungvari GS. Clinical and social determinants of long-term use of benzodiazepines and its impact on the quality of life of Chinese schizophrenia patients. Pharmacopsychiatry 2007;40:269-74.

4. Paterniti S, Dufouil C, Alperovitch A. Long-term benzodiazepine use and cognitive decline in the elderly: the epidemiology of vascular aging study. J Clin Psychopharmacol 2002;22:285-93.

5. Kamath R, Kamath S. Deprescribing in India: will we ever get down to it? Eur J Hosp Pharm 2017;24:136.

6. Reeve E, Ong M, Wu A, Jansen J, Petrovic M. A systematic review of interventions to deprescribe benzodiazepines and other hypnotics among older people. Eur J Clin Pharmacol 2017;73:927-35. 